\title{
Inhibition of Rhabdomyosarcoma's metastatic behavior through downregulation of MET receptor signaling
}

\author{
Ewa Lukasiewicz'1, Katarzyna Miekus'1, Jacek Kijowski', Grazyna Drabik², \\ Miroslawa Wilusz ${ }^{1}$, Sylwia Bobis-Wozowicz ${ }^{1}$, Marcin Majka ${ }^{1}$
}

\author{
${ }^{1}$ Department of Transplantation, Polish-American Institute of Pediatrics, Jagiellonian University \\ Medical College, Cracow, Poland \\ ${ }^{2}$ Department of Clinical Immunology, Polish-American Institute of Pediatrics, Jagiellonian University \\ Medical College, Cracow, Poland
}

\begin{abstract}
Rhabdomyosarcoma (RMS) is a soft tissue sarcoma usually diagnosed in children. In advanced and metastatic stages the prognosis is often poor. RMS cell lines were used for evaluation of the role of MET receptor inhibition on chemotaxis and invasion. In vivo studies were performed using NOD-SCID xenograft model. This study shows that blocking of MET expression has strong influence on metastatic behavior of RMS. MET negative cells possess a reduced potential to migrate and to invade. Downregulation of MET suppressed the ability of RMS cells to populate bone marrow. Inhibition of MET negative tumor cells engraftment into bone marrow was observed. MET negative tumors were also two to four times smaller than their wild type counterparts. Since MET receptor plays a very important role in facilitating metastasis of RMS cells, blocking of HGF-MET axis might be considered as a therapeutic option for RMS patients, at more advanced and metastatic stages.
\end{abstract}

Key words: MET receptor, rhabdomyosarcoma, bone marrow, invasion, homing, metastasis

\section{Introduction}

Rhabdomyosarcoma (RMS) is the most common pediatric soft tissue sarcoma usually diagnosed in the first decade of life [1]. RMS can be divided into four subtypes with embryonal RMS (ERMS) and alveoral RMS (ARMS) being predominant [1]. A distinguished characteristic of ARMS is an occurrence of chromosomal translocation leading to expression of fusion proteins PAX3-FKHR (t(2:13)(q35:q14)) and PAX7FKHR (t(1:13)(p36:q14)) [2,3]. Expression of these proteins has been correlated with a significantly worse prognosis of ARMS patients, seen as a higher risk of relapse and metastasis [4-7].

MET protooncogene, encoding receptor for Hepatocyte Growth Factor (HGF) has been shown to influence various aspects of cell biology. Interactions between MET and HGF are essential in physiological (e.g. embryogenesis, muscle development, bone remodeling) and pathological (e.g. tumor development) settings $[8,9]$.

Correspondence: M. Majka, Dept. of Transplantation,

Polish-American Institute of Pediatrics, Jagiellonian University

Medical College, Cracow, Poland; tel.: (+4812) 6591593,

fax.: (+4812) 6591593, e-mail: mmajka@cm-uj.krakow.pl
Over-expression of MET receptor is observed in a variety of tumor cells. Increased MET receptor expression may be a result of MET gene amplification [10], induction by other oncogenes, such as RAS [11], PAX3FKHR [7] or transiently due to hypoxia-activated transcription [12]. In transformed tissues, activation of MET by HGF triggers tumor growth and metastasis [13].

MET signaling exhibits profound effects on the invasive behavior of a variety of tumor cells by inducing production of various proteases such the urokinase-type plasminogen activator-dependent proteolytic network [14,15], matrix metalloproteinases and metalloproteinase inhibitors [15]. MET activation enhances transendothelial migration of cancer cells by modulating expression of adhesion molecules both on cancer cells and endothelium [16-19].

The role of MET receptor activation in pathogenesis of RMS has been documented. Activation of MET has been shown to influence proliferation, survival and migration of RMS cells [20,21]. Recently, inducible down-regulation of MET receptor obtained with lentivirus expressing an anti-MET short hairpin RNA has been demonstrated to significantly affect RMS cells biology, particularly cell proliferation and apop- 
tosis [22]. However, the effect of MET downregulation on metastatic behavior of RMS has not been studied in details. Thus, to better understand the role of MET receptor in metastasis of RMS we created cell lines with stably downregulation of MET receptor and studied their ability to migrate, to secrete extracellular matrix digesting enzymes, and to repopulate bone marrow in vivo.

\section{Materials and methods}

Cell lines. RMS cell lines used in this study (SMS-CTR-ERMS and RH30-ARMS) were generously provided by prof. M. Z. Ratajczak (University of Louisville, KY, USA) and were maintained in DMEM (Gibco BRL) supplemented with $10 \%$ heat-inactivated FBS (Gibco), $100 \mathrm{IU} / \mathrm{ml}$ penicillin, $10 \mathrm{mg} / \mathrm{ml}$ streptomycin (Gibco). Cells were cultured at $37^{\circ} \mathrm{C}, 5 \% \mathrm{CO}_{2}, 95 \%$ humidity. They were split twice a week with medium change.

Lentiviral vectors construction, production and in vitro transduction. The $p E N T R$ vector containing the MET- or LacZ-specific short hairpin RNA (shRNA) was recombinated with pLenti6/ BLOCK-iT ${ }^{\mathrm{TM}}$-DEST expression vector (Invitrogen). High titer lentiviral vector stock was produced in 293FT cells by transient lipofection of the pLenti6-GW/U6-shRNA and packaging plasmids pLP1, pLP2, and pLP/VSVG. RMS cells were transduced directly with viral supernatants and subsequently selected with blasticidin. RMS cells transduced with anti MET shRNA were indexed with shMET, while those transduced with anti LacZ - with shLacZ label.

Cell Proliferation assessment by MTT Assay. The MTT assay was performed according to the manufacturer's recommendations (Promega). Briefly, cells were seeded in 96-well plates at 104/well in $100 \mu \mathrm{l}$ of DMEM medium containing 10\% FBS. After 24, 48 and 72 hours, $10 \mu \mathrm{l}$ of CellTiter 96 Aqueous One Solution reagent were added to each well and plates were incubated for 3-4 hours. Subsequently, plates were read at $490 \mathrm{~nm}$ using the ELx800 Universal Microplate Reader (Bio-tek) and analyzed with KC4 v3.0 with PowerReports software (Bio-tek). The experiment was done two times.

Western blot. RMS cells were lysed on ice in M-Per lysing buffer (Pierce) containing protease and phosphatase inhibitors (Sigma). Subsequently, the extracted proteins were separated on a $12 \%$ sodium dodecyl sulfate-polyacrylamide electrophoresis (SDS-PAGE) gel and fractionated proteins were transferred into a PVDF membrane (BioRad). The membrane was incubated with $1 \%$ BSA for 1 hour and then overnight with primary antibodies anti-MET 1:1000 clone sc-161 (Santa Cruz Biotech.) and GAPDH as a control 1:10000 clone 2118 (Cell Signaling, Danvers, Massachusetts, USA). Subsequently proteins were detected with horseradish peroxidase (HRP)-conjugated goat anti-mouse or anti-rabbit IgG secondary antibody (Santa Cruz Biotech). The membranes were developed with an enhanced chemiluminescence (ECL) reagent (Amersham Life Sciences), dried and subsequently exposed to the HyperFilm (Amersham Life Sciences). An equal loading was evaluated by reprobing with an anti GAPDH antibody (Santa Cruz Biotech).

Chemotaxis assay. The directional movement of cells towards HGF gradient was evaluated using modified Boyden's chamber with $8 \mu \mathrm{m}$ pore polycarbonate membrane inserts (Costar Transwell; Costar-Corning). Cells were detached with $0.25 \%$ trypsin, washed and suspended in DMEM containing $0.5 \%$ BSA and seeded into the upper chamber of an insert at a density of $2.5 \times 10^{4}$ cells in $100 \mu \mathrm{l}$. The lower chamber was filled with pre-warmed medium containing HGF $(20 \mathrm{ng} / \mathrm{ml})$. 0.5\% BSA DMEM medium was used as a negative control. After 24 hours, inserts were removed from the transwells, cells were fixed with methanol. Cells which did not migrate were scraped off with cotton wool from the upper membrane and cells that had transmigrated to the lower side of the membrane were stained with Wright solution and counted under high power field (HPF) with an inverted microscope. Five fields were counted each time and the mean number of cells per HPF was calculated. The experiments were done twice in duplicates.

"Wound healing" assay. For the wound healing experiment, cells were seeded in 6 well plates and allowed to grow to complete confluence. Subsequently, a plastic pipette tip was used to scratch the cell monolayer to create a clear area, and the wounded cell layer was washed with fresh medium to remove loose cells. The cells were then maintained in DMEM containg $0.5 \%$ BSA with or without HGF $(20 \mathrm{ng} / \mathrm{ml})$ or in DMEM with $10 \%$ FBS as a positive control. After 72 hours cells were stained with Wright solution and pictures were taken. The experiments were repeated twice.

Invasion assay. GFR Matrigel invasion inserts (BD Biosciences) were rehydrated with DMEM for 2 hours and transferred to the wells containing medium with chemoattractant (HGF $20 \mathrm{ng} / \mathrm{ml}$ ) or medium alone (DMEM with $0.5 \%$ BSA) as a control. Cells were harvested by trypsinization, washed, resuspended in DMEM medium containing $0.5 \%$ BSA and seeded at density of $2.5 \times 10^{4}$ in 0.5 $\mathrm{ml}$ to the inside of the inserts. After 24 hours, cells that invaded the Matrigel were counted on the undersides of filters after fixation and staining with Wright solution. As a control of invasion the same number of control inserts (no GFR Matrigel coating) was applied. The experiments were carried out twice in duplicates.

RNA Extraction and Reverse Transcription. Total RNA was extracted using RNeasy Mini Kit (Qiagen) followed by DNAse treatment (Promega). The reverse polymerase transcription was performed using MMLV reverse transcriptase (Invitrogen) according to manufacturer's protocol.

Murine models. Short-term assay was performed as described previously [23]. Briefly, $5 \times 10^{6} \mathrm{SMS}-\mathrm{CTR}$ and $5 \times 10^{6} \mathrm{RH} 30$ cells were injected supraorbitaly into 6-8 weeks NOD-SCID mice, treated a day before with $300 \mathrm{cGy}$. Each experimental group comprised of 3-5 animals and the experiments were repeated twice. After 24 hours the mice were killed and bone marrow cells from two legs of each mouse were harvested. The cells from each leg were kept separately and total RNA was isolated using RNAeasy Kit (Qiagen). The reverse polymerase transcription was performed using MMLV reverse transcriptase (Invitrogen). The level of RMS cells homing to bone marrow was evaluated by Real-Time PCR using human GAPDH specific primers-probe set (Hs99999905 m1; Applied Biosystems). The control experiment showed no cross-reactivity with murine GAPDH.

For long term-assay $5 \times 10^{6} \mathrm{RMS}$ cells were injected subcutaneously into 6-8 weeks NOD-SCID mice. Each experimental group comprised of 3-5 animals and experiments were repeated twice. After 30 days mice were killed, tumors were harvested and were weighted.

Statistical analysis. Statistical analysis was performed using a one-way non-paired Student's t-test with Microsoft Excel.

\section{Results \\ Creation of MET negative cell lines}

We have tested two different siRNA - MET\#1 and MET\#2, and efficient inhibition of MET expression (more than $80 \%$ ) was obtained at both mRNA and pro- 
A
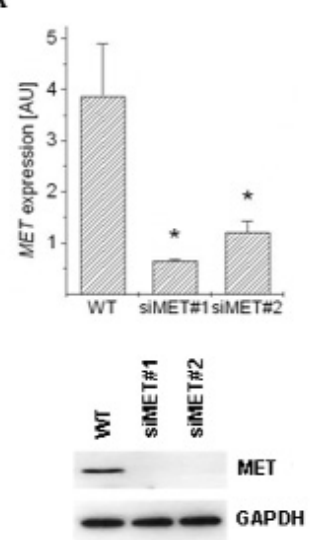

B
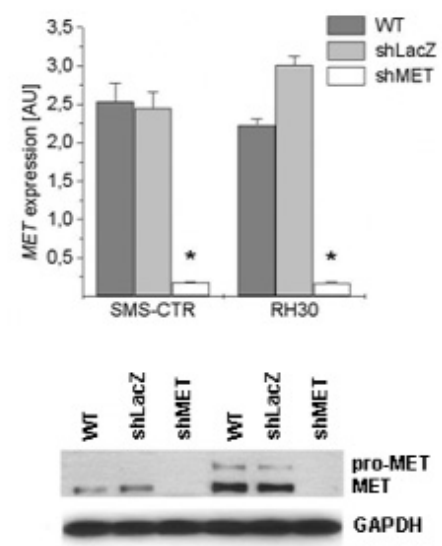

Fig. 1. Creation of MET negative RMS cell lines. Efficiency of MET downregulation was analyzed at mRNA (A and B - upper panels) and protein levels (A and B - lower panels). Cells transiently transfected with MET siRNA (A) or stable transduced with MET shRNA containing virus (B) showed almost complete downmodulation of MET receptor expression, ${ }^{*} \mathrm{p}<0.05$.

tein level (Fig. 1A). Based on these results MET shRNA was created using MET\#1 sequence and cloned into a pLenti6-GW/U6-shRNA vector. Subsequently, RMS cells were transduced with MET shRNA expressing virus. We observed over $80 \%$ downregulation of MET expression both at the mRNA and protein level in transduced cells. As a control of lacZ shRNA expressing cell lines were created that possessed similar MET receptor expression to wild type cells (Fig. 1B).

\section{Proliferation and morphology of MET negative cells}

To study the influence of MET downregulation on RMS proliferation, cells were cultured in the presence of FBS and growth curve was generated. We did not observe any differences between proliferation rate of RMSWT (wild-type; non-transfected) and RMSshMET (transduced with lentivirus) cell lines (Fig. 2A). However, serum-deprived conditions caused small but noticeable changes in morphology of RH30 $0^{\text {shMET }}$ cells which became more elongated (Fig. 2B).

\section{MET negative cells possess decreased potential to migrate and to invade extracellular matrix in vitro}

Since cell migration and invasion are important parts of metastatic behavior, we examined how inhibition of MET expression influenced these processes. First, direct migration towards HGF gradient was evaluated using modified Boyden chambers. RMS cells with downregulated MET expression were almost completely unresponsive to stimulation with HGF gradi-
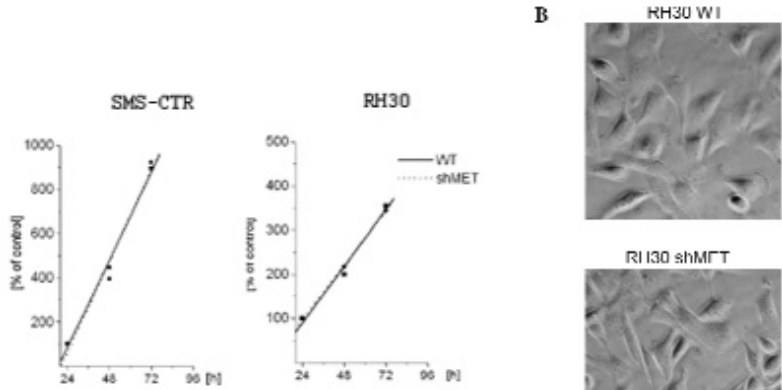

RI I30 ShMrT

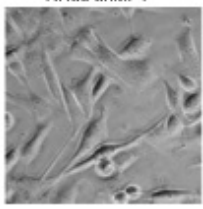

Fig. 2. Influence of MET downregulation on proliferation and morphology of RH30. ownregulation of MET receptor did not influence growth of SMS-CTR shMET and RH30shMET cells (A). RH30 3 shMET cells cultured in serum deprived conditions showed small morphological changes (pointed by arrows) not observed in wild type cells (B). Experiments were done two times.

ent. At the same time, control cells showed strong migratory response to HGF (Fig. 3A).

Since the ability to migrate through extracellular matrix $(E C M)$ is inseparable feature of metastatic tumors, we studied how silencing of HGF-MET signaling affects the potential of RMS cells to invade through matrigel coated Boyden chamber inserts. We observed complete inhibition of both SMS-CTR ${ }^{\text {shMET }}$ and RH30shMET cells ability to cross through the matrigel (Fig. 3B). This was paralleled by downregulation of mRNA expression for a protease responsible for degrading ECM. We noticed that RMSshMET cells had three to five folds lower expression of MMP9 in comparison to control cells (Fig. 3C).

Using "wound healing" assay we checked the motility of RMS cells in response to HGF. RMSshMET cells cultured in the presence of HGF did not show any motile response in contrary to RMSWT cells, which responded rapidly to HGF treatment. However, both RMSWT and RMS ${ }^{\text {shMET }}$ cells possessed similar ability to migrate when cultured in 10\% FBS (Fig. 3D).

\section{Reduced ability of ShMET cells to engraft into bone marrow and to grow in vivo}

To evaluate homing of RMSshMET tumors into bone marrow cavities, we injected them into NOD-SCID mice, and as a control, we used wild type RMS cells. After 24 hours, bone marrow from two legs was harvested and presence of human cells in the murine bone marrow was estimated using qRT-PCR. A significant decrease (two to four fold) in seeding efficiency of RMSshMET tumors was observed (Fig. 4A).

$5 \times 10^{6}$ MET - negative and - positive cells were injected s.c. into NOD-SCID mice and their ability to grow was evaluated after 30 days. Significantly, the decreased tumor size of both SMS-CTR ${ }^{\text {shMET }}$ and 

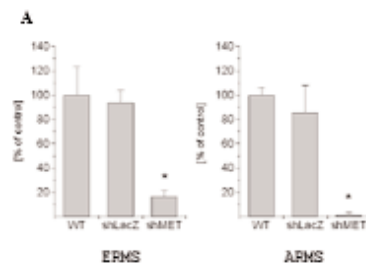

B
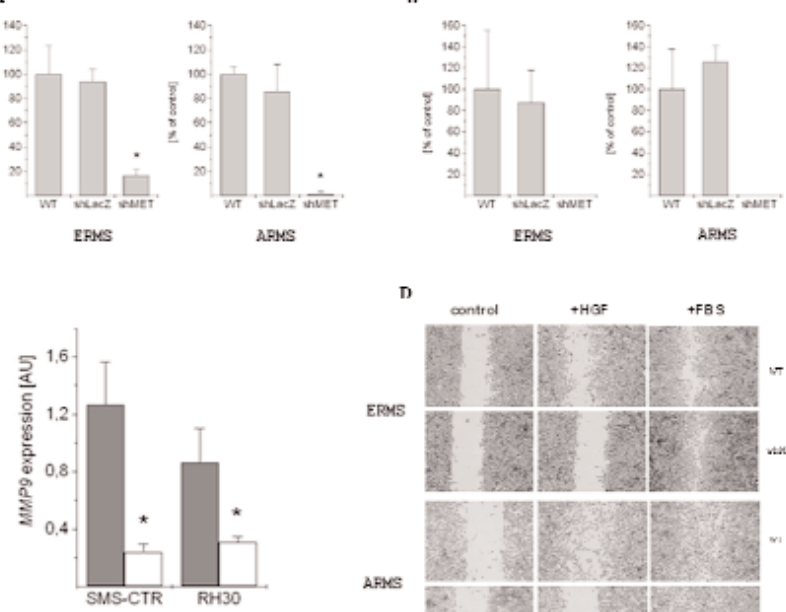

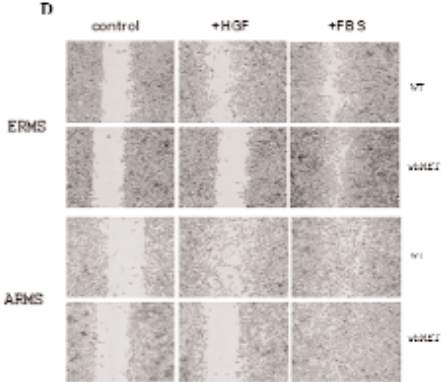

Fig. 3. MET downregulation decreases HGF-dependent overall motility and invasive behavior of RMS cells in vitro. Directional movement of RMS cells toward HGF (20 ng/ml) were studied using modified Boyden's chambers (A). Both ERMS and ARMS cell lines showed strong migratory dependence on the presence of MET receptor as both SMS-CTR ${ }^{\text {shMET }}$ and RH30 ${ }^{\text {shMET }}$ cells lines possessed only very weak chemotactic activity toward HGF in comparison to controls, $* \mathrm{p}<0.05$. Experiment was repeated two times in duplicate. HGF-mediated invasion of RMS cells was completely abolished in shMET expressing cell lines as measured by their ability to migrate through GFR Matrigel invasion inserts (B). Expression of invasion-related genes was measured in RMS cells cultured for 72 hours in a presence of HGF. Downregulation of MMP9 expression was observed (C) (control - grey bars, shMET - open bars), ${ }^{*} \mathrm{p}<0.05$. MET positive and MET negative cells were grown to confluence, scratched and stimulated with $20 \mathrm{ng} / \mathrm{ml}$ HGF or $10 \%$ FBS (D). Only control cells had the ability to migrate and close the "wound" when stimulated with HGF. However, both WT and shMET cells were equally responsive to FBS treatment. Experiment was repeated twice with similar results.

RH30shMET cells was noticed (Fig. 4B) with RMSshMET tumors being on average two to four times smaller than RMSWT tumors.

\section{Discussion}

In this study, we have shown that inhibition of MET expression by RNA interference has strong influence on metastatic behavior of RMS cells both in vitro and in vivo. Thus, we further confirmed the importance of MET receptor in the biology of RMS tumors [20-22].

Using RNAi we created RMS cell lines of both ERMS and ARMS subtypes lacking MET receptor expression as assessed by qRT-PCR and western blotting and determined their potential to migrate, invade and secrete ECM degrading enzymes in vitro and to grow and metastasize into bone marrow in vivo.

HGF-MET axis has been shown to stimulate proliferation of RMS cells whereas blocking of the axis inhibited this effect [22]. However, in our study we did
A
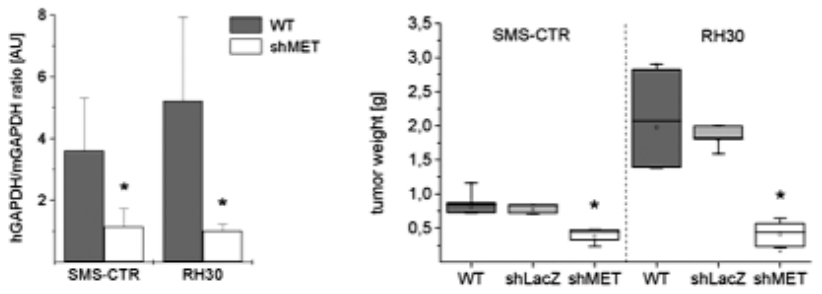

Fig. 4. In vivo homing and growth of RMS cells is dependent on MET expression. NOD-SCID model of human RMS was used to study the role of MET receptor in facilitating metastatic behavior of RMS cells. Bone marrow involvement was studied in short-term assay by injecting $5 \times 10^{6}$ tumor cells supraorbitaly into sublethaly irradiated NOD-SCID mice and harvesting bone marrow 24 hours later. Presence of human cells was calculated using qRT-PCR and human GAPDH primer set (A). Tumor growth of both ERMS shMET cells and ARMS shMET was studied by injecting $5 \times 10^{6}$ tumor cells subcutaneously and measuring the tumor weight 30 days later $(\mathbf{B})$. Decrease in both seeding efficiency and tumor size of RMSs ${ }^{\text {hMET }}$ cells was seen when compared to controls, $* \mathrm{p}<0.05$.

not observe any influence of MET downregulation on mitogenic potential of RMS cells. We tested RMS shMET cells growth and we found that proliferation rate of RMSshMET cell lines was similar to RMSWT counterparts. However, small changes in RMSshMET cells morphology in serum-free conditions were observed. The discrepancy between these two studies could be due to different culture conditions or differences in a way of MET downregulation (constitutive inhibition in the present study versus inducible inhibition in study by Taulli and coworkers [22]).

MET receptor activates the motility of RMS cells, one of the features responsible for invasive phenotype of RMS [21,24]. Using RMSshMET cells we showed that blocking of MET expression completely inhibits transmigration of both ERMS and ARMS cells through ECM, which had been shown previously [22]. However, in the previous study the mechanisms responsible for this action had not been examined. In the current study we evaluated the expression of matrix metaloproteinases responsible for degrading ECM. We noticed a strong reduction in MMP9 expression in RMSshMET cells in comparison to the wild type cells.

Jankowski and co-workers postulate that HGFMET axis may play an important role in RMS bone marrow metastasis [21]. Thus, the ability of MET negative RMS cells to populate bone marrow, one of the primary sites of RMS metastasis [1], was studied in this work. We provided here the direct proof that MET receptor is an important factor facilitating bone marrow engraftment of RMS cells.

In this study, we also confirmed and extended findings that MET downregulation blocks in vivo growth 
of both ARMS and ERMS cells. MET negative tumors were on average two times smaller than their wild type counterparts. It might derive from the lower number of blood vessels in these tumors. This phenomena is still under investigation.

In conclusion, MET receptor plays an exceptionally important role in facilitating metastasis of RMS cells and also blocking of HGF-MET axis could be used in the future as a potential therapeutic option to treat RMS patients at more advanced and metastatic stages.

Acknowledgments: This study was supported by research grant from the Polish Ministry of Science and Higher Education (N N401 229734 and N N401 010036 ), grant from Jagiellonian University Medical College (WL/292/P/L and K/ZBW/000143) and The Adam Krzyzanowski Scholarship Found.

\section{References}

[1] Parham DM, Barr FG. Skeletal muscle tumors. In: Fletcher CDM, Unni KK, Mertens F, ed. Pathology and genetics of tumors of soft tissue sarcoma. Lion: IARC press; 2002, pp. 141-154.

[2] Barr FG, Galili N, Holick J, Biegel JA, Rovera G, Emanuel BS. Rearrangement of the PAX3 paired box gene in the paediatric solid tumour alveolar rhabdomyosarcoma. Nat Genet. 1993;3:113-117.

[3] Davis RJ, D'Cruz CM, Lovell MA, Biegel JA, Barr FG. Fusion of PAX7 to FKHR by the variant $\mathrm{t}(1 ; 13)(\mathrm{p} 36 ; \mathrm{q} 14)$ translocation in alveolar rhabdomyosarcoma. Cancer Res. 1994;54:2869-2872.

[4] Galili N, Davis RJ, Fredericks WJ et al. Fusion of a fork head domain gene to PAX3 in the solid tumour alveolar rhabdomyosarcoma. Nat Genet. 1993; 5:230-235.

[5] Barr FG. Molecular genetics and pathogenesis of rhabdomyosarcoma. J Pediatr Hematol Oncol. 1997;19:483-491.

[6] Sorensen PH, Lynch JC, Qualman SJ et al. PAX3-FKHR and PAX7-FKHR gene fusions are prognostic indicators in alveolar rhabdomyosarcoma: a report from the children's oncology group. J Clin Oncol. 2002;20:2672-2679.

[ 7] Ginsberg JP, Davis RJ, Bennicelli JL, Nauta LE, Barr FG. Upregulation of MET but not neural cell adhesion molecule expression by the PAX3-FKHR fusion protein in alveolar rhabdomyosarcoma. Cancer Res. 1998;58:3542-3546.

[ 8] Lesko E, Majka M. The biological role of HGF-MET axis in tumor growth and development of metastasis. Front Biosci. 2008;13:1271-1280.

[9] Jiang W, Hiscox S, Matsumoto K, Nakamura T. Hepatocyte growth factor/scatter factor, its molecular, cellular and clinical implications in cancer. Crit Rev Oncol Hematol. 1999;29: 209-248.

[10] Lutterbach B, Zeng Q, Davis LJ et al. Lung cancer cell lines harboring MET gene amplification are dependent on Met for growth and survival. Cancer Res. 2007;67:2081-2088.

[11] Ivan M, Bond JA, Prat M, Comoglio PM, Wynford-Thomas D. Activated ras and ret oncogenes induce over-expression of c-met (hepatocyte growth factor receptor) in human thyroid epithelial cells. Oncogene. 1997;14:2417-2423.

[12] Pennacchietti S, Michieli P, Galluzzo M, Mazzone M, Giordano S, Comoglio PM. Hypoxia promotes invasive growth by transcriptional activation of the met protooncogene. Cancer Cell. 2003;3:347-361.

[13] Jeffers M, Rong S, Anver M, Vande Woude GF. Autocrine hepatocyte growth factor/scatter factor-Met signaling induces transformation and the invasive/metastastic phenotype in C127 cells. Oncogene. 1996;13:853-856.

[14] Nishimura K, Matsumiya K, Miura H et al. Effects of hepatocyte growth factor on urokinase-type plasminogen activator (uPA) and uPA receptor in DU145 prostate cancer cells. Int $J$ Androl. 2003;26:175-179.

[15] Gong R, Rifai A, Tolbert EM, Centracchio JN, Dworkin LD. Hepatocyte growth factor modulates matrix metalloproteinases and plasminogen activator/plasmin proteolytic pathways in progressive renal interstitial fibrosis. J Am Soc Nephrol. 2003; 14:3047-3060.

[16] Hiscox S, Jiang WG. Regulation of endothelial CD44 expression and endothelium-tumour cell interactions by hepatocyte growth factor/scatter factor. Biochem Biophys Res Commun. 1997;233:1-5.

[17] Mine S, Fujisaki T, Kawahara C et al. Hepatocyte growth factor enhances adhesion of breast cancer cells to endothelial cells in vitro through up-regulation of CD44. Exp Cell Res. 2003;288:189-197.

[18] Trusolino L, Cavassa S, Angelini P et al. HGF/scatter factor selectively promotes cell invasion by increasing integrin avidity. FASEB J. 2000;14:1629-1640.

[19] Kawakami-Kimura N, Narita T, Ohmori K et al. Involvement of hepatocyte growth factor in increased integrin expression on HepG2 cells triggered by adhesion to endothelial cells. $\mathrm{Br}$ J Cancer. 1997;75:47-53.

[20] Sharp R, Recio JA, Jhappan C et al. Synergism between INK4a/ARF inactivation and aberrant $\mathrm{HGF} / \mathrm{SF}$ signaling in rhabdomyosarcomagenesis. Nat Med. 2002 8:1276-1280.

[21] Jankowski K, Kucia M, Wysoczynski M, Reca R et al. Both hepatocyte growth factor (HGF) and stromal-derived factor-1 regulate the metastatic behavior of human rhabdomyosarcoma cells, but only HGF enhances their resistance to radiochemotherapy. Cancer Res. 2003;63:7926-7935.

[22] Taulli R, Scuoppo C, Bersani F et al. Validation of met as a therapeutic target in alveolar and embryonal rhabdomyosarcoma. Cancer Res. 2006;66:4742-4749.

[23] Lesko E, Gozdzik J, Kijowski J, Jenner B, Wiecha O, Majka M. HSP90 antagonist, geldanamycin, inhibits proliferation, induces apoptosis and blocks migration of rhabdomysoracoma cells in vitro and seeding into bone marrow in vivo. AntiCancer Drugs. 2007;18:1173-1181.

[24] Rees H, Williamson D, Papanastasiou A et al. The MET receptor tyrosine kinase contributes to invasive tumour growth in rhabdomyosarcomas. Growth Factors. 2006;24: 197-208.

Submitted: 26 May, 2009 Accepted after reviews: 20 June, 2009 